\title{
Sensitivity experiments of a severe rainfall event in north-western Italy: 17 August 2006
}

\author{
M. Milelli ${ }^{1}$, E. Oberto ${ }^{1}$, and A. Parodi ${ }^{2}$ \\ ${ }^{1}$ Regional Agency for Environmental Protection - Arpa Piemonte, Torino, Italy \\ ${ }^{2}$ CIMA Research Foundation, Savona, Italy
}

Received: 19 December 2007 - Revised: 25 March 2008 - Accepted: 28 April 2008 - Published: 9 July 2008

\begin{abstract}
This study is embedded into a wider project named "Tackle deficiencies in Quantitative Precipitation Forecast (QPF)" in the framework of the COSMO (COnsortium for Small-scale MOdelling) community. In fact QPF is an important purpose of a numerical weather prediction model, for forecasters and customers. Unfortunately, precipitation is also a very difficult parameter to forecast quantitatively. This priority project aims at looking into the COSMO Model deficiencies concerning QPF by running different numerical simulations of various events not correctly predicted by the model. In particular, this work refers to a severe event (moist convection) happened in Piemonte region during summer 2006. On one side the results suggest that details in orography representation have a strong influence on accuracy of QPF. On the other side COSMO Model exhibits a poor sensitivity on changes in numerical and physical settings when measured in terms of QPF improvements. The conclusions, although not too general, give some hint towards the behaviour of the COSMO Model in a typical convective situation.
\end{abstract}

\section{Introduction}

The main goal of this project was to gain a deeper understanding of the COSMO Model problems in terms of QPF. A list of test cases has been prepared by the Meteorological Services of the participating countries (Germany, Switzerland, Italy, Poland, Greece and Romania) on the basis of the known problems of the model in the different regions of interest. To ensure that those problems were not due to an old version of the model or a specific implementation, the test cases were rerun with a COSMO Model reference version. The test cases, for which the COSMO Model reference version reproduced the QPF deficiencies, constituted a final list of 25 test cases (about 4 for each of the countries) recommended for sensitivity studies (Dierer et al., 2007). Eventually a list of sensitivity studies concerning data assimilation, numerics, physics and initial conditions was prepared and applied to each test case. For the sake of brevity the complete lists of test cases and sensitivity studies cannot be reported here (see Milelli et al., 2007). The list of sensitivity studies includes about 20 modifications that were performed for all dates included in the final list of test cases, plus a few

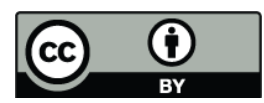

Correspondence to: M. Milelli (massimo.milelli@arpa.piemonte.it) cross experiments performed in a second step (see Table 1) on which we focus in this work. Globally, more than 600 simulations have been performed.

The test case here described considers a severe rainfall event, which affected the Northern part of Piemonte, at the border with Switzerland, during 17-18 August 2006. The event was mainly convective in nature and characterized by the occurrence of intense deep moist convection leading to relevant rainfall depths. The results of the numerical experiments are compared in terms of peak and mean rainfall depths over the catchments both for short duration $(6 \mathrm{~h}$, not shown here, see Milelli et al., 2007) and long duration (24 h). The analysis of the results helps in giving a more precise idea about the model parameters, about what parts of the model need to be reformulated in order to improve the QPF and about the scales which can be actually solved by the model. This work will focus on some of the most relevant simulations.

\section{Event analysis}

The analysis of the atmospheric scenario at the synoptic scale highlights the presence of an intense cyclonic circulation during the event. On August 17 00:00 UTC, the upper air trough $(500 \mathrm{hPa})$ was localized near the Biscaglia gulf and during

Published by Copernicus Publications. 


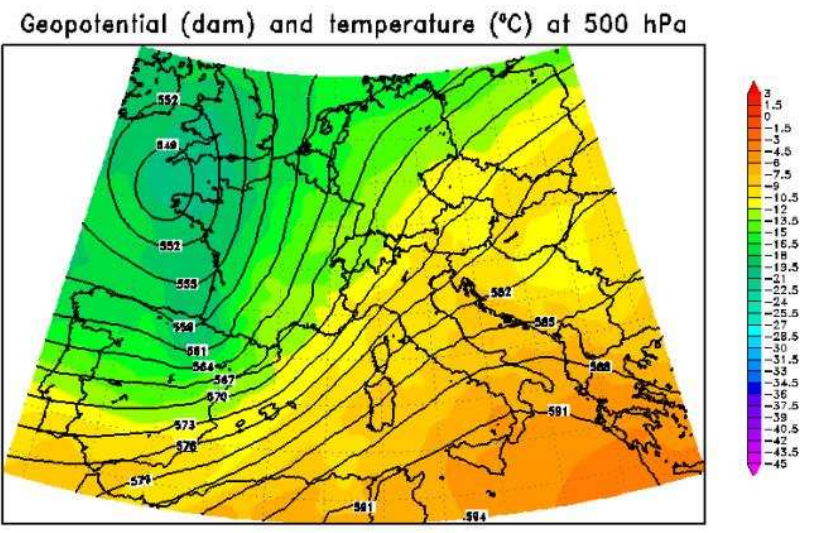

ECMWF_EURCM_0250 - Thu 17 AUG 2006 18:00 UTC - Anolysis

Figure 1. ECMWF analysis of $500 \mathrm{hPa}$ Geopotential (solid lines) and temperature (colors) for 17 August, 18:00 UTC.

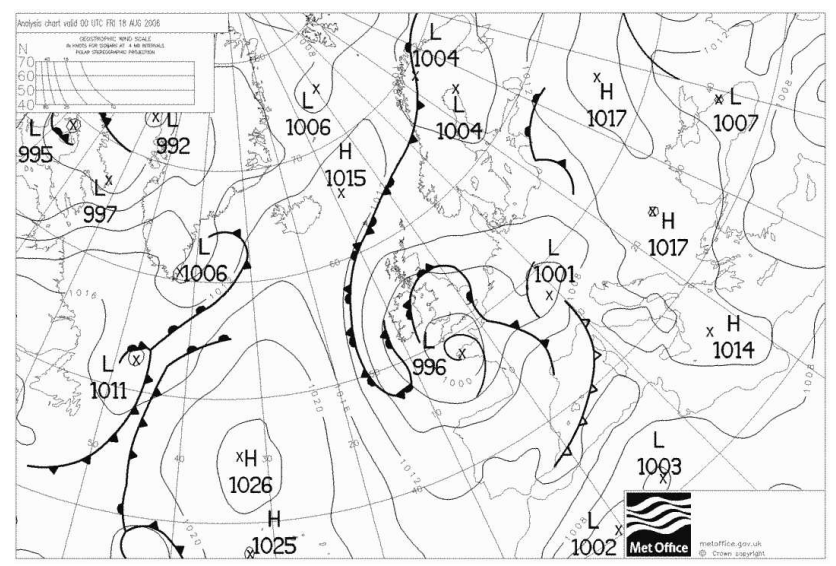

Figure 2. UKMO-Bracknell analysis chart for 17 August, 18:00 UTC.

the afternoon the trough moved gradually towards the Atlantic French coastline (ARPA Piemonte, 2006). Meanwhile, the anticyclonic ridge continued its expansion over Eastern Europe; this structure triggered an intensification of strong baric gradient between Western and Eastern areas of Europe and consequently an increase of large-scale advection of moist and unstable air over Northern Italy and in particular in the area of north-western Alps (Fig. 1).

The flux of moist air continued for the whole day and during the evening the upper-level cold front crossed Piemonte region (Fig. 2) intensifying deep moist convection in complex orography areas.

This large-scale meteorological configuration, typical of late summer season, is known to induce extreme rainfall events in the area of the north-western Italy (Rudari et al., 2004). Looking at the spatial distribution of the observed rainfall depths from rain gauges of the ARPA Piemonte high-
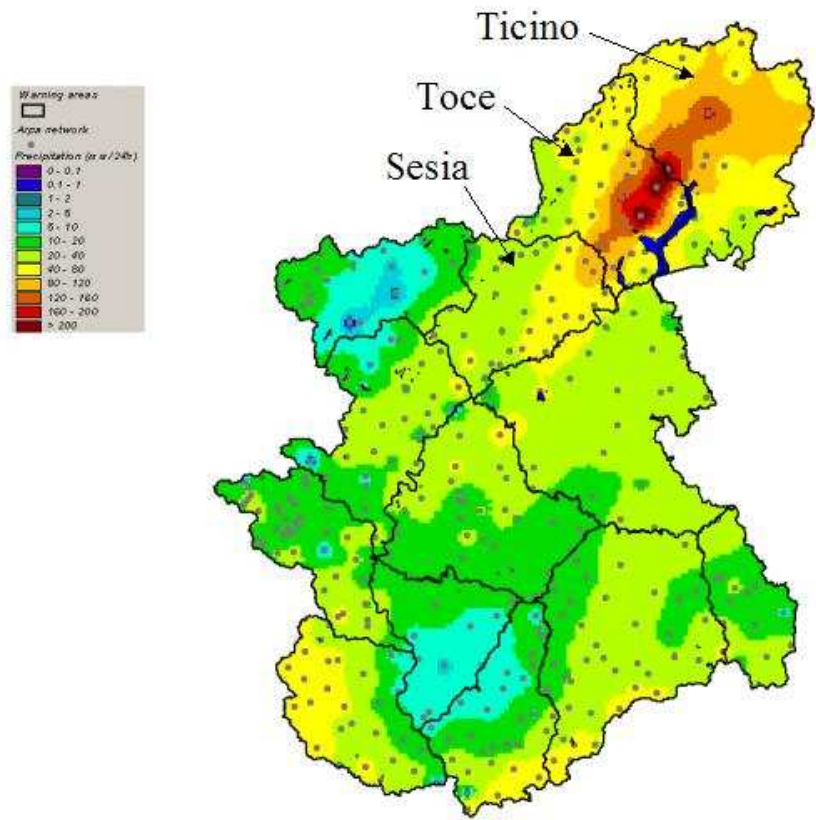

Figure 3. Observed rainfall depths in the north-western Italy. The panel refers to the $24 \mathrm{~h}$ cumulated rainfall depth (06:00 UTC 17 August-06:00 UTC 18 August). The rain gauges are plotted in grey and the warning areas in black. The affected areas, indicated with arrows, are Ticino $(\mathrm{CH})$, Toce and, marginally, Sesia.

resolution network (Fig. 3), the big role of the orography is easily recognised. Indeed, the most intense part of the event occurred in the areas characterized by steep and high orography, perpendicular to the dominant advection direction from South-West to North-East.

\section{The COSMO Model}

The COSMO Model is a non-hydrostatic limited-area atmospheric model developed within the COSMO community for applications on the meso- $\beta$ and meso- $\gamma$ scale (Steppeler et al., 2003). The model is based on non-hydrostatic, fully compressible hydro-thermodynamical equations in advection form. Generalized terrain-following height coordinates with rotated geographical coordinates are used. The model equations are solved on an ARAKAWA C-grid with user-defined vertical grid staggering. They are spatially discretised with second-order finite differences. Time integration uses a 2nd order leapfrog (horizontally explicit, vertically implicit) time-split integration scheme including extensions proposed by Skamarock and Klemp (1992). A 4th order linear horizontal diffusion is calculated. 3-dimension divergence damping and off-centering are applied in split time steps. Damping at the top of the model domain is done by Rayleigh damping (see for instance Doms and Schättler, 2002) in the upper layers. Data at the lateral boundaries are 


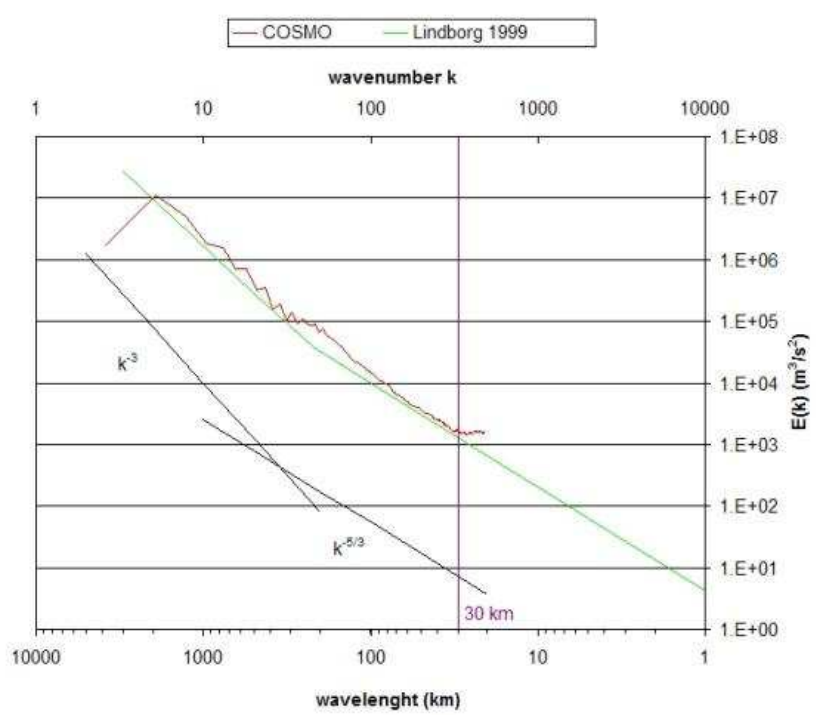

Figure 4. Turbulence spectrum of the COSMO Model forecast (in red), compared to the theoretical spectrum obtained by Lindborg (1999) (in green). The -3 and $-5 / 3$ slopes are also indicated (in black). The two lines diverge at $\approx 4 \Delta x$.

prescribed using a Davies-type one-way nesting (see Davies, 1976 or Davies , 1983).

Subgrid-scale turbulence is parameterized by a prognostic turbulent kinetic energy closure at level 2.5 including effects from subgrid-scale condensation and from thermal circulations (Mellor and Yamada, 1974). The surface layer parameterisation is based on turbulent kinetic energy and includes a laminar-turbulent roughness layer. The formation of precipitation is described by a bulk microphysics parameterisation including water vapour, cloud water and ice, rain and snow with a fully prognostic treatment of precipitation, i.e. three-dimensional transport of rain, and snow is calculated. Condensation and evaporation are parameterized by saturation adjustment while depositional growth/sublimation of cloud ice is calculated using an explicit non-equilibrium growth equation. Subgrid-scale cloudiness used for radiation calculations is parameterized by an empirical function depending on relative humidity, ice content and height. Moist convection is parameterized using a mass-flux scheme with an equilibrium closure based on moisture convergence following Tiedtke (1989). Radiation is calculated using a twostream scheme for short- and longwave fluxes (eight spectral intervals) including a full cloud-radiation feedback. A multilayer version of the soil model solving the heat conduction equation is applied.

\section{Description of the sensitivity studies}

Before defining the different model configurations, it is important to point out that all of these experiments have been performed switching off the data assimilation procedure.
Table 1. List of the cross experiments.

\begin{tabular}{ll}
\hline Name & Characteristics \\
\hline A1 & Ctrl \\
A2 & Ctrl with Runge-Kutta and 10\% reduction in initial humidity \\
A3 & Ctrl with Kain-Fritsch/Bechtold convection scheme \\
A4 & A2 with Kain-Fritsch/Bechtold convection scheme \\
A5 & Ctrl with modified Tiedtke scheme \\
A6 & A2 with modified Tiedtke scheme \\
A7 & Ctrl with Runge-Kutta and 10\% increase in initial humidity
\end{tabular}

Sensitivity experiments are divided in studies regarding initial conditions, numerical methods and physical parameterizations. An overview of sensitivity experiments and expected changes can be found in Dierer et al. (2007) or in Milelli et al. (2007).

Concerning the Piemonte event, the results vary according to the area of averaging (as it will be shown later) but in general, the QPF of COSMO Model at $7 \mathrm{~km}$ shows a certain insensitivity with respect to change in its physical and numerical formulation. Moreover, the conclusions are similar for different accumulation periods ( 6 or $24 \mathrm{~h}$, not shown here). When compared to the observed mean rainfall depths, most of the experimental settings provide quite satisfactory results $(+/-10 \%$ with respect to the control run) with the exception of the values given by the runs with different initial humidity conditions (2 runs), different microphysics (2 runs) and different convection schemes ( 2 runs) in which the precipitation is sensibly reduced. It has to be stressed that the area of average is about $8500 \mathrm{~km}^{2}$ and corresponds to the area of the three affected zones (Sesia, Toce and Ticino).

Different conclusions arise if the peak rainfall depths are considered: in this case most of the experimental runs reveal a strong underestimation of the observed peak values with a shift of the maximum towards the Ticino area. The deficiency of COSMO Model at $7 \mathrm{~km}$ in reproducing the orographically triggered convection is not surprising and seen for other weather forecast models at horizontal resolution of the order of $10 \mathrm{~km}$. In this framework, Lin et al. (2001) have recently synthesized a few common synoptic and mesoscale scenarios prone to heavy orographic rainfall based on the study of some US, Alpine and East Asian test cases. They single out the following common synoptic and mesoscale ingredients leading to heavy orographic rainfall: a) conditionally or potentially unstable air stream impinging on the mountains, b) a very moist low-level jet, c) a steep mountain and d) quasi-stationary synoptic system slowing down the convective system over the threatened area. Among them, a) and c) are recognized to be the most important factors and certainly contributed to the occurrence of extreme observed rainfall. The unstable nature of the air stream affecting the North-Eastern mountainous area of Piemonte is confirmed by the analysis of soundings located in CuneoLevaldigi and Milano-Linate (not shown here). Moreover, 


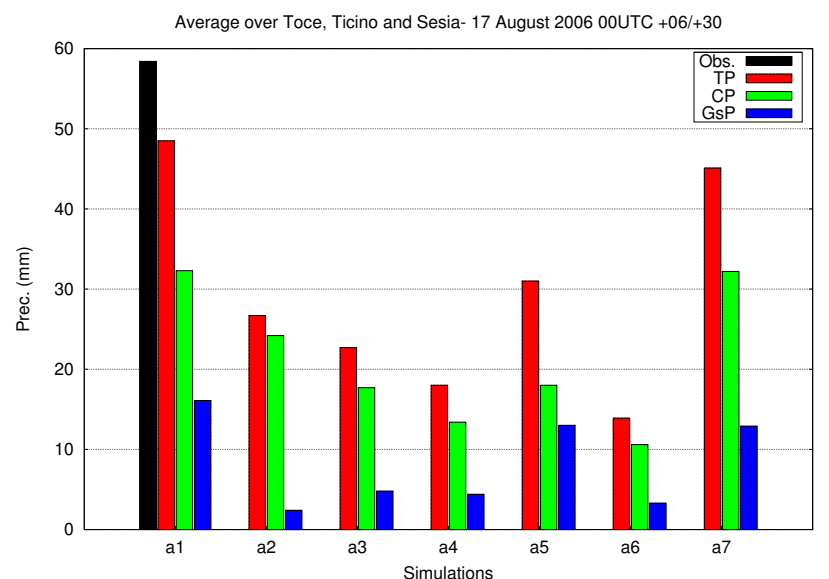

Figure 5. Total Precipitation in the $+6 \mathrm{~h} /+30 \mathrm{~h}$ time interval for the different runs (see Table 1 for the description of the simulations). Total Precipitation in red (TP), Convective Precipitation in green (CP), Grid-scale Precipitation in blue (GsP) and observations in black. Average performed over the affected areas.

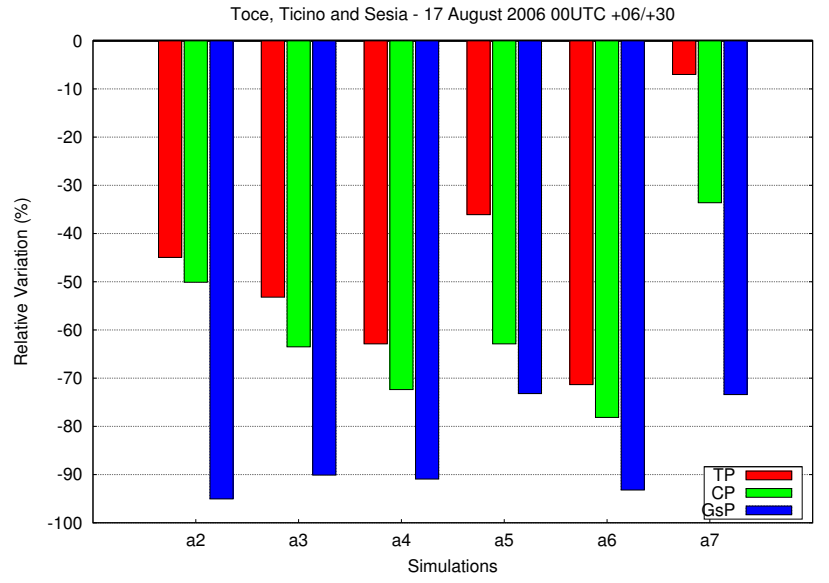

Figure 6. Relative bias $(\%)$ of the different simulations with respect to the Ctrl run (A1) for different domains in the $+6 \mathrm{~h} /+30 \mathrm{~h}$ time interval (see Table 1 for the description of the runs). Average performed over the affected areas. Total Precipitation in red (TP), Convective Precipitation in green (CP) and Grid-scale Precipitation in blue (GsP).

the highest rainfall peaks were observed in a relief system having an average elevation of $2000 \mathrm{~m}$ and a spatial width of order $10-20 \mathrm{~km}$. It seems clear that this model resolution is unable to capture the small-scale orography variability proper of that area. In fact the theoretical horizontal resolution is around $7 \mathrm{~km}$ but the effective one is $\approx 30 \mathrm{~km}$ as shown in (Fig. 4). This value corresponds roughly to $4 \Delta x$ and agrees with the values found in literature (see for instance Skamarock, 2004). Therefore, the simulated air mass

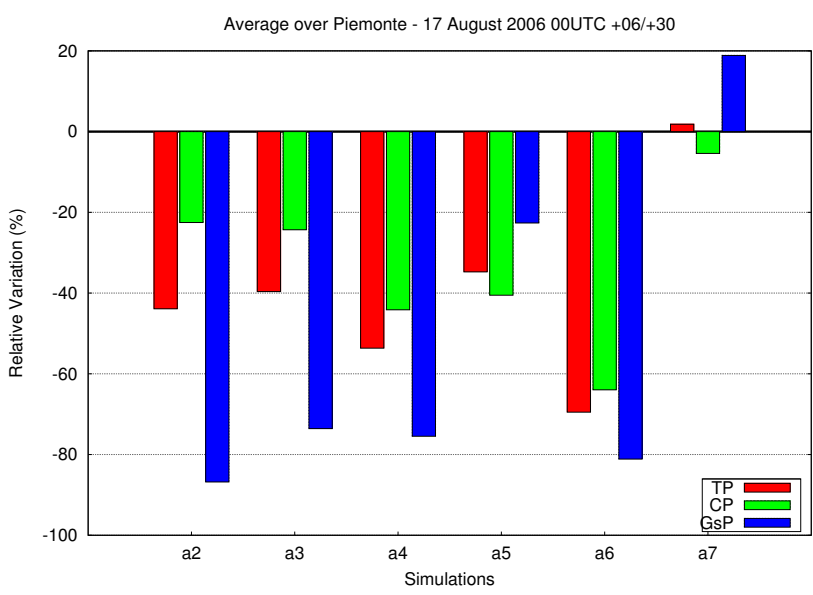

Figure 7. Relative bias (\%) of the different simulations with respect to the Ctrl run (A1) for different domains in the $+6 h /+30 \mathrm{~h}$ time interval (see Table 1 for the description of the runs). Average performed over Piemonte region. Total Precipitation in red (TP), Convective Precipitation in green $(\mathrm{CP})$ and Grid-scale Precipitation in blue (GsP).

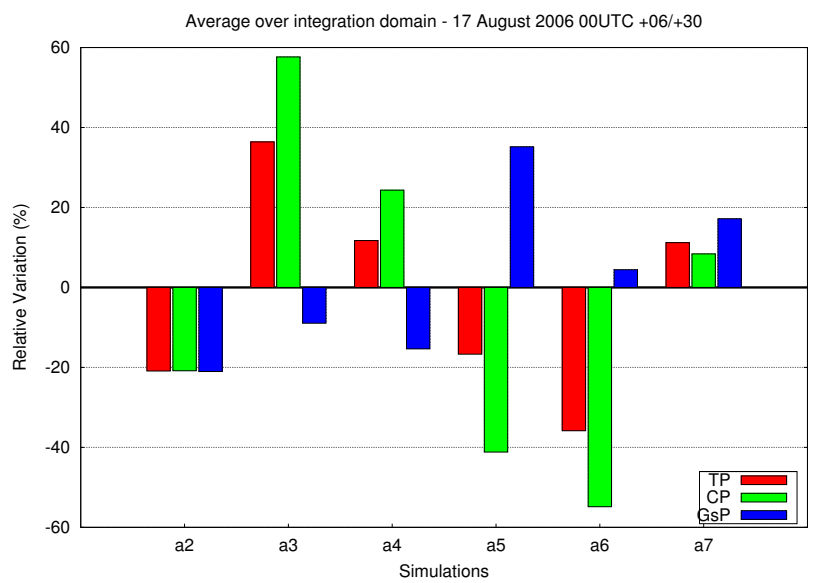

Figure 8. Relative bias (\%) of the different simulations with respect to the Ctrl run (A1) for different domains in the $+6 \mathrm{~h} /+30 \mathrm{~h}$ time interval (see Table 1 for the description of the runs). Average performed over the whole domain of the simulation. Total Precipitation in red (TP), Convective Precipitation in green (CP) and Grid-scale Precipitation in blue (GsP).

does not "see" correctly these orography structures: the convection dynamics cannot be properly triggered and lee-side effect is missing in the simulation. A more detailed analysis of the results is described in Milelli et al. (2007).

\subsection{Cross experiments}

In a second step of the project, cross experiments have been considered. They are performed using the latest version of 
the COSMO Model. This version is differing from the reference version used in the previous simulations mainly by microphysics changes in the warm rain scheme and changes of snow physics. The cross experiments are described in Table 1 .

The changes are related to the convection scheme, to the initial humidity conditions and to the dynamics. In detail:

- the following convection schemes are tested: Tiedtke (Tiedtke, 1989), Kain-Fritsch/Bechtold (Bechtold et al., 2001) (see also Kain and Fritsch $(1990,1993)$ ) and a modified Tiedtke scheme with changes related to evaporation, turbulent entrainment, mixed-phase saturation adjustment and exchange of cloud water and cloud ice with grid-scale variables;

- the initial conditions have been modified: $10 \%$ increase and $10 \%$ decrease of humidity;

- differences in the dynamics have been introduced: three time-level Leapfrog scheme with time-split treatment of acoustic and gravity waves and Runge-Kutta scheme.

The results in Fig. 5 demonstrate that there is indeed a sensitivity to these changes: the decrease of initial humidity together with Runge-Kutta scheme produces a relative reduction of precipitation (A1 vs. A2, A3 vs. A4, A5 vs. A6), while the increase of humidity is almost balanced by Runge-Kutta scheme with an overall reduction (A1 vs. A7). Comparing the effect of the cross experiments with reduced initial humidity and Runge-Kutta scheme with their single effects leads to the conclusion that they are simply summing up (Dierer et al., 2007).

The bias in Figs. 6, 7 and 8, calculated with respect to the the Ctrl run (A1) shows that the area where the verification is performed may vary the results. In fact, it is evident that if we average over a larger area (Figs. 7 and 8), the initial humidity increase plays a more important role, giving an increase of precipitation in A7 with respect to A1. Moreover there is a disagreement between the relative effect of runs A3 and A4 in case we average over Piemonte region (Fig. 7) or over the whole domain of the simulation (Fig. 8). In the latter case these runs show a relative increase of precipitation. The situation is more evident when we compare directly the difference between the total precipitation cumulated in $24 \mathrm{~h}$ of A3 and A1 (Fig. 9). It is evident that there is an overall increase of total precipitation, but locally some structures are completely missing in run A3. Looking at the structures over Belgium and Eastern France, there seems to be a shift of the precipitation line, since we observe two parallel stripes of overestimation and underestimation, but over Piemonte we just have an evident lack of convective cells. It has to be pointed out that the precipitation in Figs. 6, 7 and 8 has been calculated averaging over the grid points falling into the three warning areas of Ticino, Toce and Sesia (see Fig. 3, about $8500 \mathrm{~km}^{2}$ ), over the Piemonte and Ticino area (see Fig. 3,

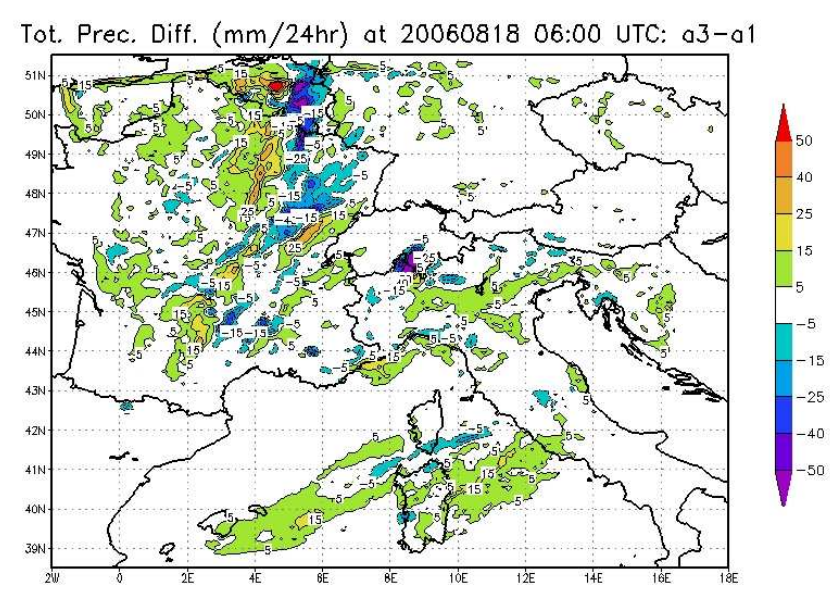

Figure 9. Total Precipitation difference in the $+6 \mathrm{~h} /+30 \mathrm{~h}$ time interval (06:00 UTC 17 August-06:00 UTC 18 August) between A3 and A1 (see Table 1 for the description of the runs).

about $35000 \mathrm{~km}^{2}$ ) and over the whole domain of the simulation (see Fig. 9) respectively. The reason for the behaviour of runs A3 and A4 could be found in the characteristics of the Kain-Fritsch/Bechtold scheme (Bechtold et al., 2001): here the closure assumption is based on CAPE and the onset of convection depends on the large-scale vertical velocity (in the Tiedtke scheme the closure assumption is based on moisture convergence and convection is triggered if the parcel's temperature exceeds the environment temperature by a fixed temperature threshold of $0.5 \mathrm{~K}$, see Tiedtke (1989)). This determines an overestimation of the average precipitation over the plains and a reduction of the maxima over the mountains, with respect to the Tiedtke scheme. Consequently, by enlarging the averaging domain, more and more flat regions are included and this counterbalance the underprediction over the Alps which is the dominant effect when considering only a part of the simulation area (Figs. 6 and 7).

\section{Conclusions}

Sensitivity runs have been carried out for a single test case (heavy convective precipitation event over Piemonte region on 17 August 2006) in order to look for the relative changes. A selection of most representative simulations has been chosen, in particular concerning cross experiments. Although there is no clear suggestion about an improvement of the control simulation, because the main problem is the general underestimation of the peaks due to subgrid unresolvable effects (orography) and no physical parameterisation or numerical scheme can cure this effect, some indication can be here highlighted:

- poor sensitivity of the model concerning average values, with some exception, but not in the desired direction 
(initial humidity, microphysics, convection schemes);

- in particular Kain-Fritsch/Bechtold scheme seems to spread (and increase) the precipitation over the domain, but the peaks over complex orography are smoothed;

- the validation results (average values) vary according to the considered domain.

It has to be pointed out that this is a single test case, but the results are in agreement with the other cases included in the study by other COSMO members. This kind of study will continue inside the COSMO community, with further investigations. In particular, as far as the convection is concerned, it could be worth performing simulations at a higher horizontal resolution, using scales where the deep convection should be resolved by the model itself in an explicit way.

Acknowledgements. The authors wish to thank Renata Pelosini and Franco Siccardi for their support, their colleagues at ARPA Piemonte and the colleagues of the COSMO community for the constructive collaboration. A special thank to Silke Dierer for the coordination of the project. We are also grateful to two anonymous referees for the valuable comments.

Edited by: F. Stel and D. B. Giaiotti

Reviewed by: two anonymous referees

\section{References}

ARPA Piemonte: Rapporto sull'evento meteopluviometrico del 1618 Agosto 2006, Internal Report, 2006 (in Italian).

Bechtold, P., Bazile, E., Guichard, F., Mascart, P., and Richard, E.: A mass flux convection scheme for regional and global models, Q. J. Roy. Meteor. Soc., 127, 869-886, 2001.

Davies, H. C.: A lateral boundary formulation for multi-level prediction models, Q. J. Roy. Meteor. Soc., 102, 405-418, 1976.

Davies, H. C.: Limitations of some common lateral boundary schemes used in regional NWP models, Mon. Weather Rev., 111, 1002-1012, 1983.

Dierer, S., Arpagaus, M., Damrath, U., Seifert, A., Achimowicz, J., Avgoustoglou, E., Andreadis, T., Baldauf, M., Dumitrache, R., Fragkouli, V., Grazzini, F., Louka, P., Mercogliano, P., Mezzasalma, P., Milelli, M., Mironov, D., Morgillo, A., Oberto, E., Parodi, A., Pescaru, I. V., Pflueger, U., Schubiger, F., Starosta, K., and Tesini, M. S.: Final report of the COSMO priority project "Tackle deficiencies in quantitative precipitation forecasts”, COSMO Internal Report, 1-47, 2007.
Doms, G. and Schättler, U.: A description of the non-hydrostatic regional model LM. Part I: Dynamics and Numerics, http://www.cosmo-model.org/content/model/documentation/ core/default.htm, 1-140, November 2002.

Kain, J. S. and Fritsch, J. M.: A one-dimensional entraining/detraining plume model and its application in convective parameterization, J. Atmos. Sci., 47, 2784-2802, 1990.

Kain, J. S. and Fritsch, J. M.: Convective parameterization for mesoscale models: The Kain-Fritsch scheme. The representation of cumulus convection in numerical models, Meteor. Monogr., 27, Amer. Meteor. Soc., 165-170, 1993.

Lin, Y. L., Chiao, S., Wang, T., Kaplan, M., and Weglarz, R.: Some Common Ingredients for Heavy Orographic Rainfall, Weather Forecast., 16(6), 633-660, 2001.

Lindborg, E.: Can the atmospheric kinetic energy spectrum be explained by two-dimensional turbulence?, J. Fluid Mech., 388, 259-288, 1999.

Mellor, G. L. and T. Yamada, T.: A hierarchy of turbulence closure models for planetary boundary layers, J. Atmos. Sci., 31, 17911806, 1974.

Milelli, M., Oberto, E., and Parodi, A.: Proscenium Project official report, 1-24, 2007.

Rudari, R., Entekhabi, D., and Roth, G.: Terrain and Multiple Scale Interactions as Factors in Generating Extreme Precipitation Events., J. Atmos. Sci., 5(3), 390-404, 2004.

Skamarock, W. C. and Klemp, J. B.: The Stability of Time-Split Numerical Methods for the Hydrostatic and the Nonhydrostatic Elastic Equations, Mon. Weather Rev., 120, 2109-2127, 1992.

Skamarock, W. C.: Evaluating Mesoscale NWP Models Using Kinetic Energy Spectra, Mon. Weather Rev., 132, 3019-3032, 2004.

Steppeler, J., Doms, G., Schaettler, U., Bitzer, H. W., Gassmann, A., Damrath, U., and Gregoric, G.: Meso-gamma scale forecasts using the non-hydrostatic model LM, Meteorol. Atmos. Phys., 82, 75-96, 2003.

Tiedtke, M.: A comprehensive mass flux scheme for cumulus parametrization in large-scale models, Mon. Weather Rev., 117, 1779-1800, 1989. 